US Army Corps

of Engineers ${ }_{\circledast}$

Engineer Research and

Development Center

Terrain Conditions Forecasting Program

Characterizing Snow Surface Properties

Using Airborne Hyperspectral Imagery for Autonomous Winter Mobility

Taylor S. Hodgdon, Anthony J. Fuentes, Brian G. Quinn,

September 2021

Bruce C. Elder, and Sally A. Shoop
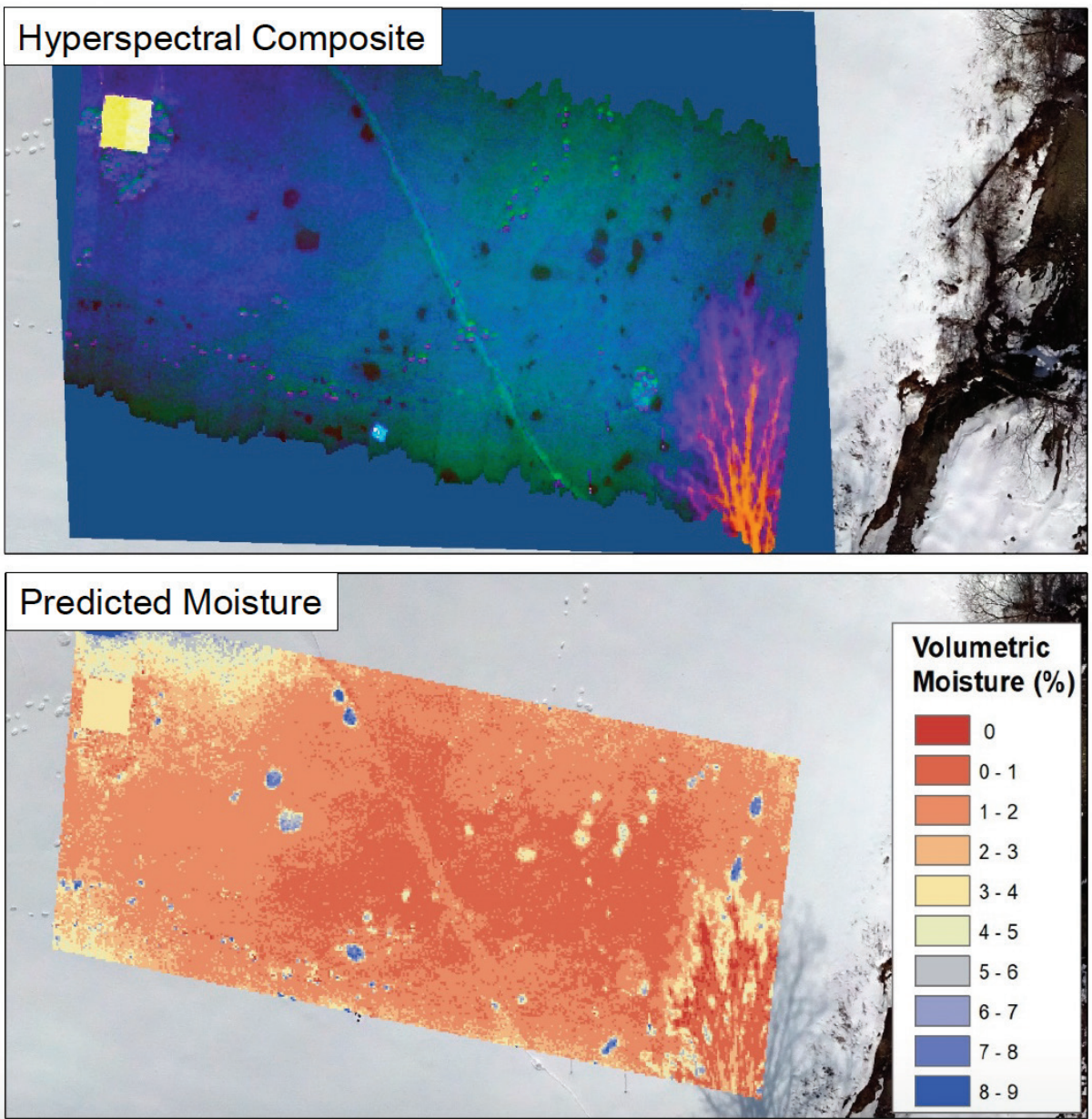

Approved for public release; distribution is unlimited. 
The U.S. Army Engineer Research and Development Center (ERDC) solves the nation's toughest engineering and environmental challenges. ERDC develops innovative solutions in civil and military engineering, geospatial sciences, water resources, and environmental sciences for the Army, the Department of Defense, civilian agencies, and our nation's public good. Find out more at www.erdc.usace.army.mil.

To search for other technical reports published by ERDC, visit the ERDC online library at https://erdclibrary.on.worldcat.org/discovery. 


\section{Characterizing Snow Surface Properties Using Airborne Hyperspectral Imagery for Autonomous Winter Mobility}

Taylor S. Hodgdon, Anthony J. Fuentes, Brian G. Quinn, Bruce C. Elder, and Sally A. Shoop

U.S. Army Engineer Research and Development Center (ERDC) Cold Regions Research and Engineering Laboratory (CRREL) 72 Lyme Road

Hanover, NH 03755-1290

Final Report

Approved for public release; distribution is unlimited.

Prepared for Headquarters, U.S. Army Corps of Engineers

Washington, DC 20314-1000

Under Work Unit 2686F6, Project number BP4, Task number SBP402, Program Element number 0603463A 


\section{Abstract}

With changing conditions in northern climates it is crucial for the United States to have assured mobility in these high-latitude regions. Winter terrain conditions adversely affect vehicle mobility and, as such, they must be accurately characterized to ensure mission success. Previous studies have attempted to remotely characterize snow properties using varied sensors. However, these studies have primarily used satellite-based products that provide coarse spatial and temporal resolution, which is unsuitable for autonomous mobility. Our work employs the use of an Unmanned Aerial Vehicle (UAV) mounted hyperspectral camera in tandem with machine learning frameworks to predict snow surface properties at finer scales. Several machine learning models were trained using hyperspectral imagery in tandem with in-situ snow measurements. The results indicate that random forest and k-nearest neighbors models had the lowest Mean Absolute Error for all surface snow properties. A Pearson correlation matrix showed that density, grain size, and moisture content all had a significant positive correlation to one another. Mechanically, density and grain size had a slightly positive correlation to compressive strength, while moisture had a much weaker negative correlation. This work provides preliminary insight into the efficacy of using hyperspectral imagery for characterizing snow properties for autonomous vehicle mobility. 


\section{Contents}

Abstract

2 Field Collections............................................................................................................... 3

2.1 Field site: Union Village Dam, Thetford............................................................... 3

2.2 In-situ physical snow properties......................................................................... 4

2.3 In-situ mechanical snow properties................................................................... 5

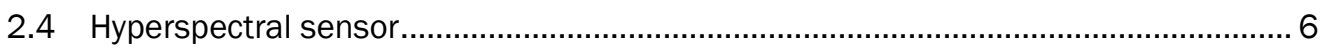

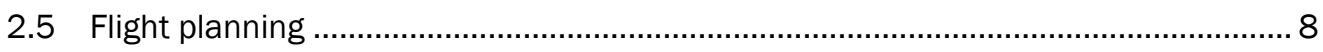

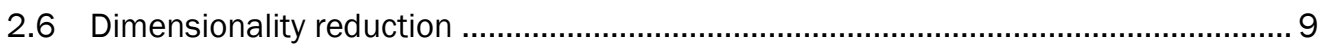

2.7 Hyperspectral data processing ..................................................................... 12

2.8 Photogrammetry and orthorectification .................................................... 13

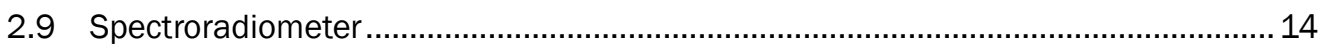

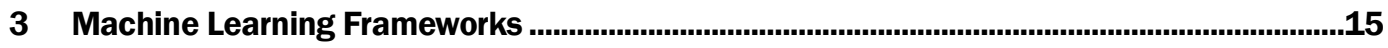

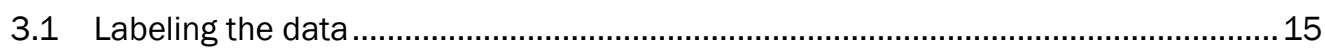

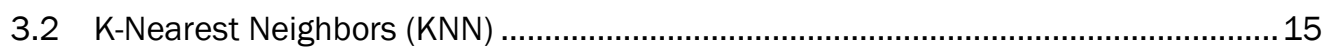

3.3 Decision trees .............................................................................................. 16

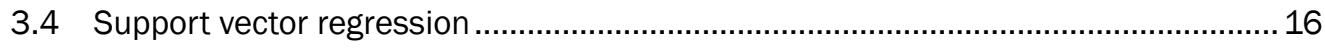

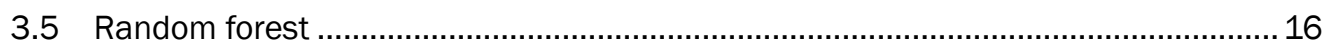

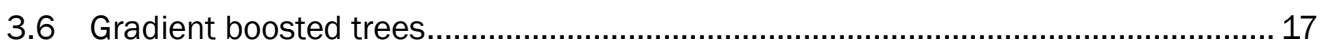

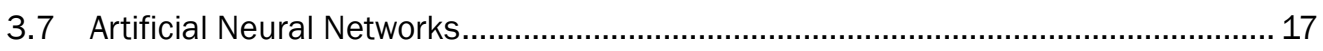

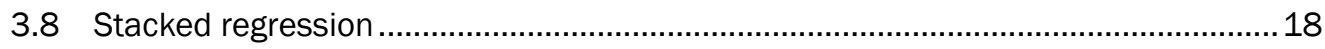

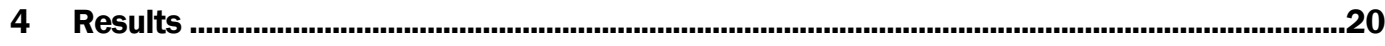

4.1 Model performance .......................................................................................... 20

4.2 Correlations between physical and mechanical measurements ............................23

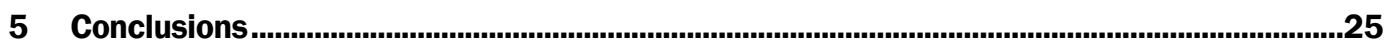

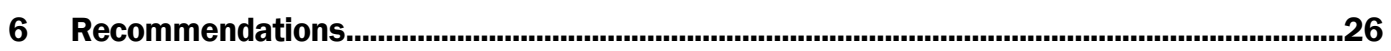

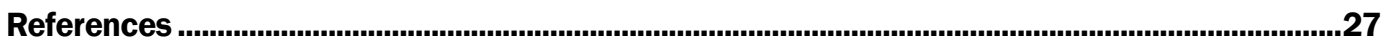

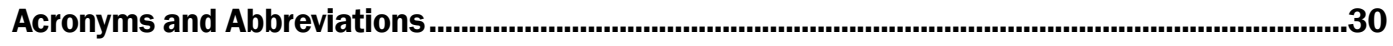

Report Documentation Page 


\section{Figures and Tables}

\section{Figures}

Figure 1. Location of the five sample sites in the northern field at Union Village Dam. Sample sites are demarcated by the red bounding boxes. For scale, the red boxes are 5 -ft $x$ 5-ft.

Figure 2. Closeup of one of the staked sample sites showing the flat topography of the northern field. The sloping southern field is in located in the background of the image.

Figure 3. Aerial image of in-situ snow measurements taking place at one of the test sites. The corner of the test site is located at the base of the wooden stake.

Figure 4. Schematic diagram showing how the friction sled was used to calculate the coefficient of friction between the sled and the snow surface.

Figure 5. Pika $L$ hyperspectral sensor mounted onto the gimbal caddy of the Infinity 9 system.

Figure 6. Results of K-means data reduction test on data collected from KRC. Each color represents a different "class" or "cluster" identified by the K-means algorithm.

Figure 7. The corrected reflectance hyperspectral data before (left) and after (right) orthorectification. For scale, the white region in the image is the calibration tarp.

Figure 8. Skyraider UAS platform with high resolution camera used for collecting photogrammetric images.

Figure 9. Using the field spectroradiometer on the white Spectralon reference plate.

Figure 10. Boxplots showing each ML model's performance (MAE) when predicting snow grain size, moisture content, and density.

Figure 11. Orthomosaic image collected on 3/11/21 when the snow had wet pockets forming near the surface; associated hyperspectral composite composed primarily of the NIR bands; and the predicted moisture using the hyperspectral false color composite as the input data into the KNN model framework.

Figure 12. Pearson correlation matrix comparing relationships between in-situ physical and mechanical snow properties.

\section{Tables}

Table 1. Example format of input text file for machine learning frameworks.

Table 2. Artificial neural network model hyperparameters. ANN Model: physical property used to train model; Input Neurons: number of nodes in input layer; Hidden Layers: number of hidden layers in each network; Neurons: number of neurons in each hidden layer. 


\section{Preface}

This study was conducted for the United States Army Corps of Engineers through the Machine Learning for Winter Mobility Forecasting Congressional Add project, part of the Terrain Conditions Forecasting Program, under Work Unit 2686F6, Project number BP4, Task number SBP402, and Program Element number 0603463A.

The work was performed by the Terrestrial and Cryospheric Sciences Branch (Dr. John Weatherly, Chief); the Engineering Resources Branch (Dr. Caitlin A. Callaghan, Chief); and the Force Projection and Sustainment Branch (Dr. Wade Lein, Acting Chief) of the Research and Engineering Division, U.S. Army Engineer Research and Development Center, Cold Regions Research and Engineering Laboratory (ERDCCRREL). At the time of publication, Dr. George Calfas was Division Chief. The Deputy Director of ERDC-CRREL was Mr. David B. Ringelberg, and the Director was Dr. Joseph L. Corriveau.

COL Teresa A. Schlosser was Commander of ERDC, and Dr. David W. Pittman was the Director. 


\section{Introduction}

\subsection{Background}

With growing tensions in northern climates, it is crucial for the United States to have assured mobility in high-latitude regions. Recently there has been growing interest in the U.S. Army for off-road autonomous vehicle capabilities. Autonomous vehicles allow for unmanned scouting missions through potentially hostile territory. While there has been increased interest in this area for on-road autonomy, significant knowledge gaps exist for highlatitude regions where there are substantial seasonal impacts on terrain (Parker et al. 2021). Winter terrain conditions, such as snow, adversely affect vehicle mobility and, as such, they must be accurately characterized to ensure mission success. Previous studies have built inference models, trained using Red, Green, Blue (RGB) imagery, that can predict different types of ground classes, such as snow, pavement, or ice specifically for vehicle mobility purposes (Hodgdon et al. 2021; Parker et al. 2021). While it's useful to be able to distinguish between different types of ground surfaces, a critical property that is missing from these analyses is the compressive strength of the underlying material, which is crucial to determine if the material will support a given vehicle. Instead of using classification frameworks to predict surface type, regression models can be constructed that predict a range of surface properties that can be used for strength characterization (Selige et al. 2006; Ewing et al. 2020). Recent studies have attempted to remotely characterize snow properties using hyperspectral and multispectral sensors (Nolin et al. 2000; Dozier and Painter 2004; Gusain et al. 2004; Shekhar et al. 2019; Haq et al. 2019; Singh et al. 2019). Some have tried predicting properties such as grain size, density, and moisture content, which are important to understand in order to predict the overall mechanical response of the snow (strength). These studies have primarily used satellite-based products that typically provide coarse spatial and temporal resolution, thereby leaving out potential information that can be ascertained from finer scales. To get finer spatial and temporal hyperspectral data, sensors can be mounted on Unmanned Aerial Systems (UAS) and flown out in front of vehicle convoys to provide look-ahead information for routing purposes. As part of this analysis, we aimed to build a framework combining trained regression algorithms with a UAS mounted hyperspectral sensor to characterize snow surface properties on spatial and temporal scales relevant for autonomous vehicle mobility. 


\subsection{Objectives}

The primary objective of this analysis was to test common regression frameworks using UAS collected hyperspectral imagery to predict physical snow surface properties for autonomous vehicle mobility. Secondarily, we wanted to collect mechanical measurements of the snow pack to see how the physical snow properties related to the overall mechanical response of the snow.

\subsection{Approach}

To test this approach, we collected airborne hyperspectral imagery in tandem with in-situ physical and mechanical snow measurements throughout the 2020-2021 winter season. Union Village Dam (UVD) in Thetford, VT, was used as the collection site as it is a facility managed by the U.S. Army Corps of Engineers, allowing for easy access. Across the field site there were five different sample locations where in-situ snow measurements were collected each day. Data were collected on five separate days with varying snow conditions to effectively capture the variability for model training. Each hyperspectral dataset was subsampled using Principal Component Analysis (PCA) to reduce the data size and minimize noise in the training data. The hyperspectral data was labeled with the corresponding in-situ snow properties and then split into training (70\%) and test (30\%) data to ensure model overfitting did not occur. The labeled data was then used to train several different regression algorithms to see which was most accurate. Detailed descriptions of the field site, collection methods, data processing, and machine learning algorithms are outlined in the Methods section of this report. In the conclusions section we discuss the results and describe which algorithms are most well-suited for characterizing physical snow properties. 


\section{Field Collections}

\subsection{Field site: Union Village Dam, Thetford}

The field site for this work was located at Union Village Dam in Thetford. The study area consisted of two large fields bisected by the Ompompanoosuc River (Figure 1). The northern field was relatively flat with very small undulations in the topography, while the southern field was primarily located on a hill slope with a $30 \%$ grade. To make sure the in-situ snow measurements spatially aligned with the correct regions of the hyperspectral data, five test plots were staked out in the northern field. Each plot was selected to provide increased variability in the snow cover due to proximity to the river and tree canopy around the edge of the field. The plots were 5 -ft x 5 -ft squares demarcated by wooden stakes with reflective tape on top (Figure 2). The reflective tape was used to clearly identify site boundaries in the hyperspectral data.

Figure 1. Location of the five sample sites in the northern field at Union Village Dam. Sample sites are demarcated by the red bounding boxes. For scale, the red boxes are $5-\mathrm{ft} \times 5$-ft.

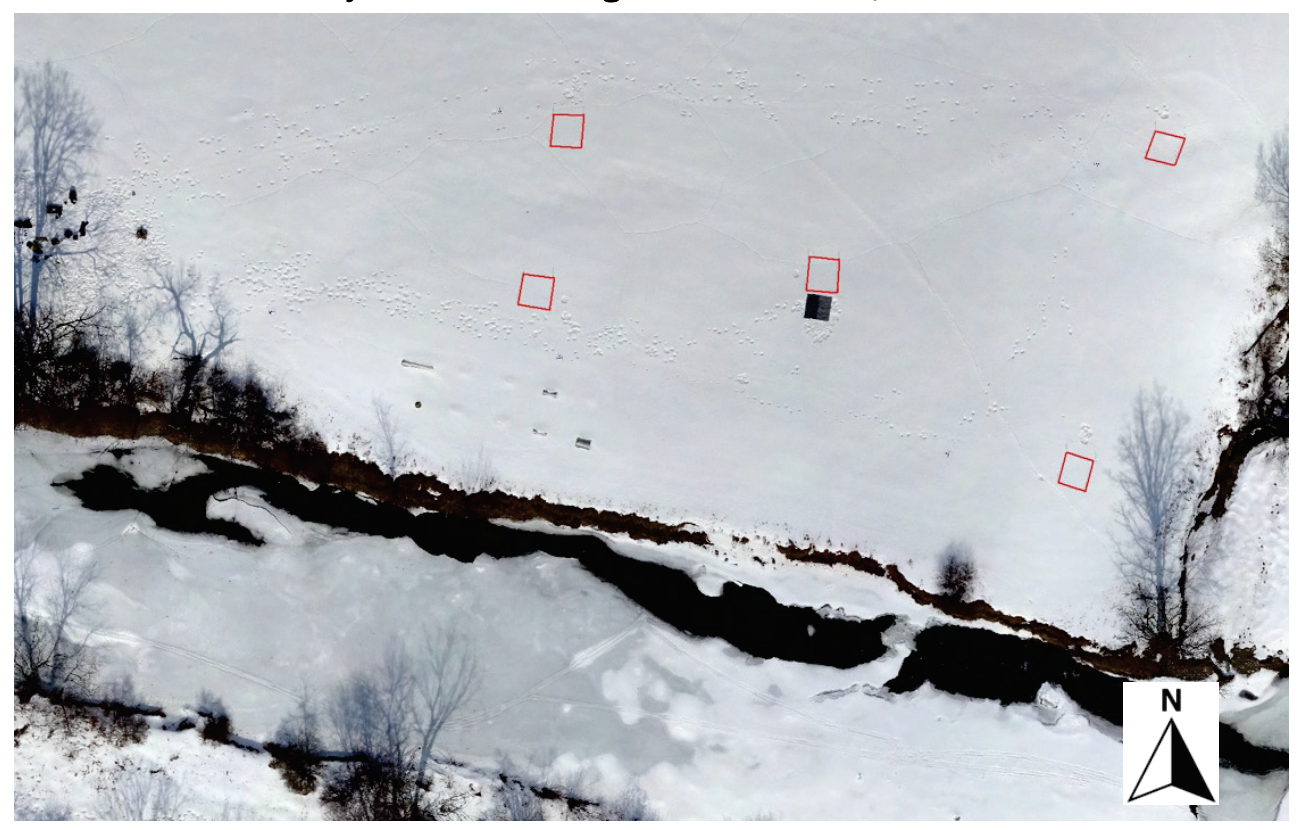


Figure 2. Closeup of one of the staked sample sites showing the flat topography of the northern field. The sloping southern field is in located in the background of the image.

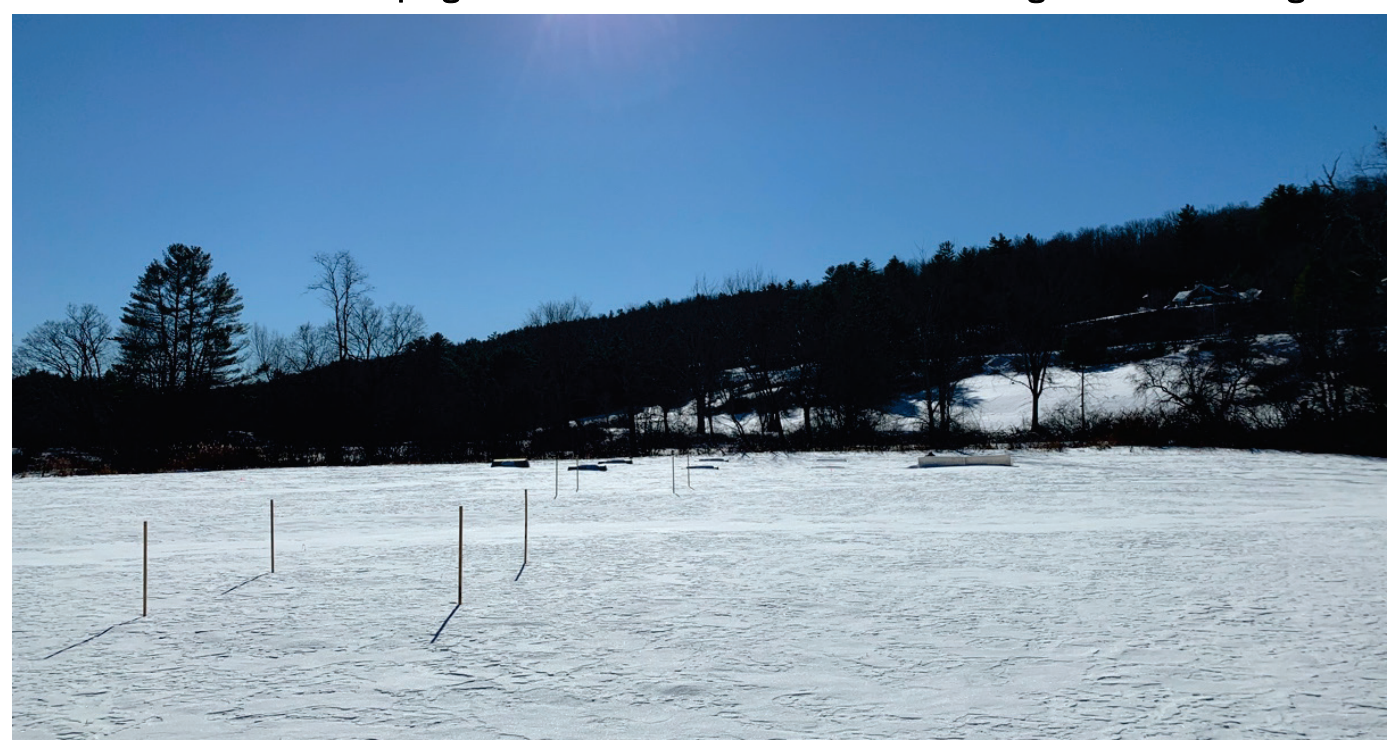

\subsection{In-situ physical snow properties}

Each day, several physical properties of the snow pack were measured at every staked site (Figure 3). Before physical properties were measured, a snow pit was dug to determine vertical profiles of snow temperature, density, and grain size. Air temperature and snow surface temperature were also measured. For the physical properties of the snow, grain size, density, and grain shape were all measured. Grain size and shape were visually determined using a hand lens and crystallography card with sizes ranging from 1-3 $\mathrm{mm}$ size. Average grain size, to the nearest $0.5 \mathrm{~mm}$ was recorded for each sample site. A $100 \mathrm{~cm}^{3}$ snow cutter was inserted into the surface layer of the snow, then the weight was determined using an electronic scale. Density was calculated from the measured weight and known volume of the cutter. Finally, moisture content was measured at each site using a Denoth moisture meter (Denoth et al. 1984). The moisture meter measures the dielectric constant of the snow, which can be used with the density to calculate the liquid water content. 
Figure 3. Aerial image of in-situ snow measurements taking place at one of the test sites. The corner of the test site is located at the base of the wooden stake.

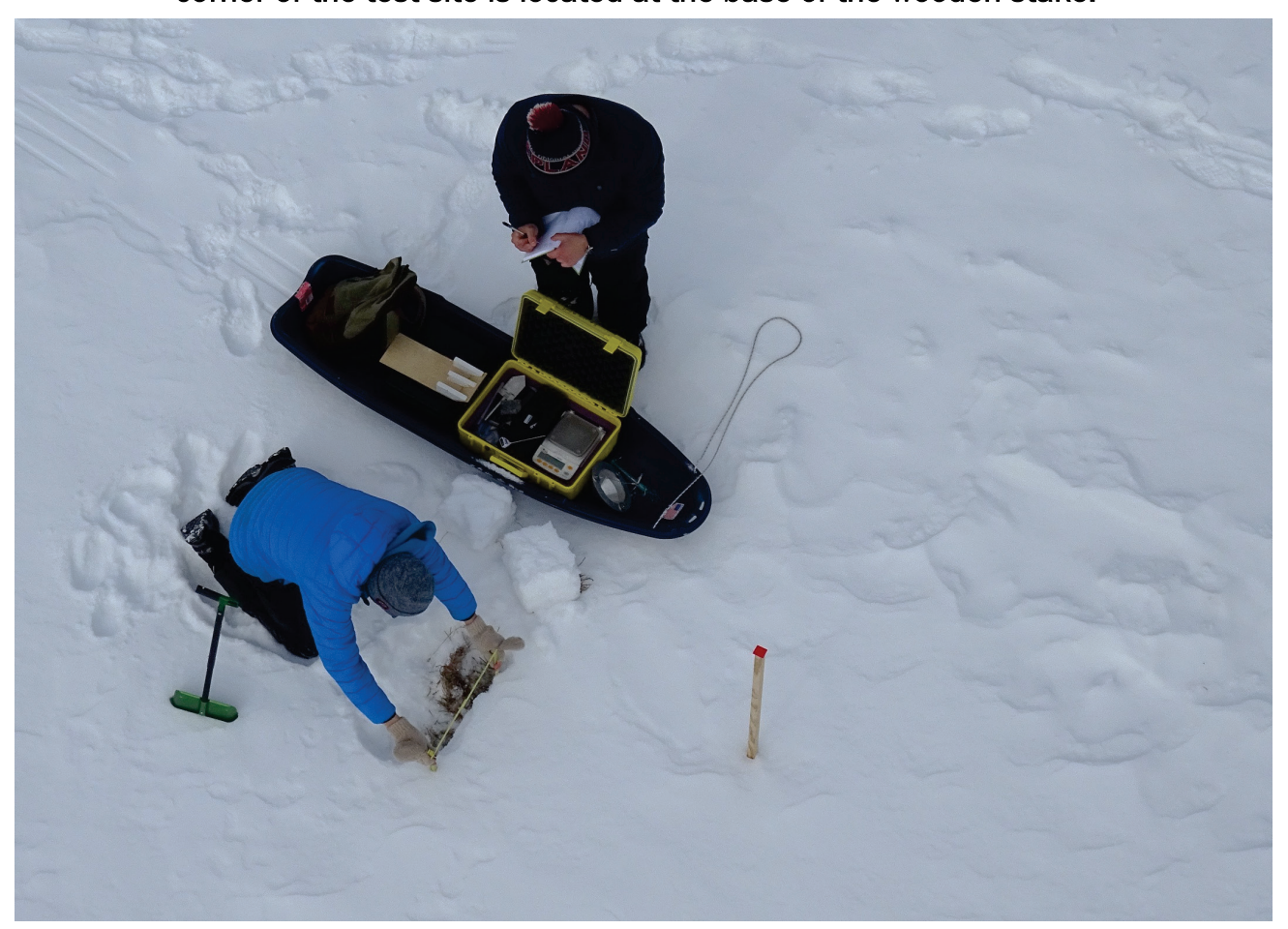

\subsection{In-situ mechanical snow properties}

In addition to the physical snow properties measured at each field site, mechanical tests were also conducted to determine the compressive strength and the surface friction of the snow. A Yamaha drop cone (Perla 1969) was used to measure compressive strength of virgin (uncompacted) snow. The drop cone was dropped from a height of $25.4 \mathrm{~cm}$ (10 in) above the snow surface. The height above the snow was determined using a meter stick, to ensure the same drop height was used for each measurement. If the cone was not level with the snow surface after the drop, the measurement was retaken. Based upon how far the cone penetrates the snow, a compressive strength can be calculated using:

$$
P=\frac{m g H}{V}
$$

Where $\mathrm{P}$ is the hardness, or compressive strength of the snow, $\mathrm{m}$ is the mass of the drop cone (1050 g), g is gravitational acceleration $\left(9.81 \mathrm{~m} / \mathrm{s}^{2}\right)$, $\mathrm{h}$ is the height from which the cone was dropped $(25.4 \mathrm{~cm})$, and $\mathrm{V}$ is the volume of snow displaced by the cone upon impact. At each site, three drop cone measurements were taken, and the average of the drops was used as the representative compressive strength for the site. 
Surface friction was also measured at each field site, quantified by the coefficient of friction between a friction sled and the snow surface. The friction sled was designed to be lightweight with a large surface area-tomass ratio so there would be little or no sinkage in the snow. The bottom of the sled was lined with a smooth butadiene rubber with a Shore 6oA hardness value to match the characteristics of rubber used in typical tires. The sled was pulled horizontally across the snow surface using a spring scale. Schematics are shown in Figure 4. The force required to move the sled was determined from the spring scale and the coefficient of friction was calculated using:

$$
\mathrm{CoF}=F_{H} / F_{N}
$$

Where CoF is the coefficient of friction between the sled and the snow, $\mathrm{F}_{\mathrm{H}}$ is the resistive force of the sled measured using the spring scale $(\mathrm{N})$, and $\mathrm{F}_{\mathrm{N}}$ is the normal force $(\mathrm{N})$ of the sled acting upon the snow (Zhang 2016).

Figure 4. Schematic diagram showing how the friction sled was used to calculate the coefficient of friction between the sled and the snow surface.

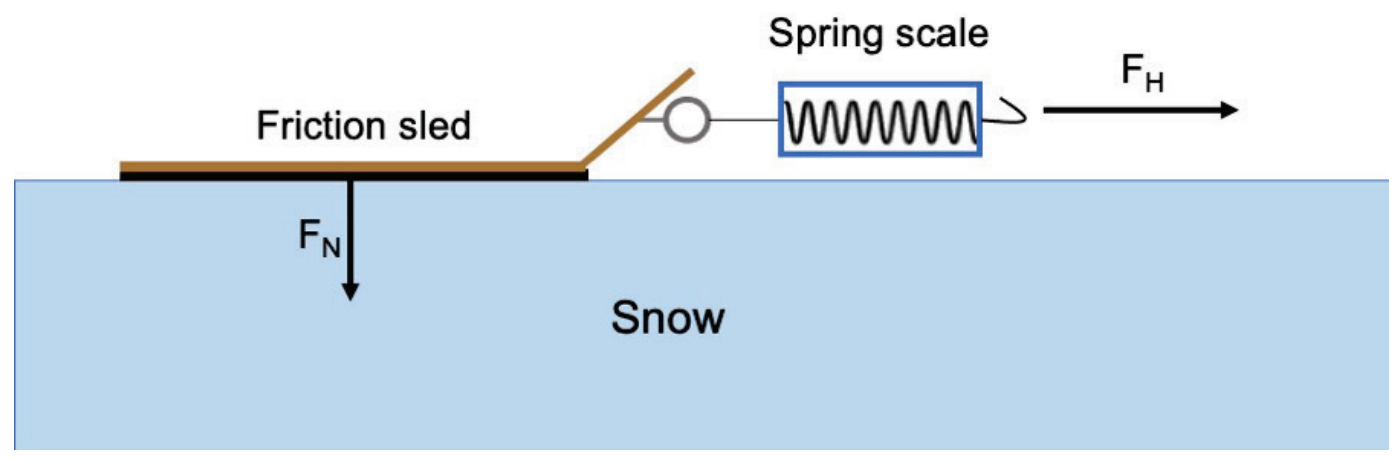

\subsection{Hyperspectral sensor}

The hyperspectral imager used in this study was a Resonon Pika L visible to near-infrared (VIS-NIR) sensor configured for UAS-based airborne collections (Figure 5). The Pika L has a spectral range of 400 to $1000 \mathrm{~nm}$ that covers the range from the visible region of the electromagnetic spectrum to the NIR. In the airborne configuration, the Pika L is limited to 150 bands to reduce the size of the measured data cubes to conserve memory space on the onboard solid-state drive. The Pika L operates as a push-broom sensor whereby a hyperspectral data cube is compiled from individual scan lines that are collected as the UAS moves forward along a 
linear flight line, analogous to the operating principle of a computer printer. Each scan line is composed of an across-track (widthwise) set of pixels that capture all 150 spectral bands for each pixel. The Pika $L$ has a fixed across track resolution of 900 pixels that is constrained by the size of the focal array of the instrument. The minimum width of each pixel is constrained by the flight altitude, lens instantaneous field of view (IFOV), and the resolution of the orthorectification digital elevation model (DEM) acquired from either aerial photogrammetry or Lidar data. Each data cube is limited to 2000 scan lines in the along-track direction (forward direction of travel) after which the flight computer saves the scan and instantiates a new scan with short period of non-collection. A $17 \mathrm{~mm}$ fixedfocus, calibrated Schneider lens with a field of view (FOV) of $17.6 \mathrm{deg}$ and an IFOV of 0.71 milliradians was used for all data collections.

Figure 5. Pika $L$ hyperspectral sensor mounted onto the gimbal caddy of the Infinity 9 system.

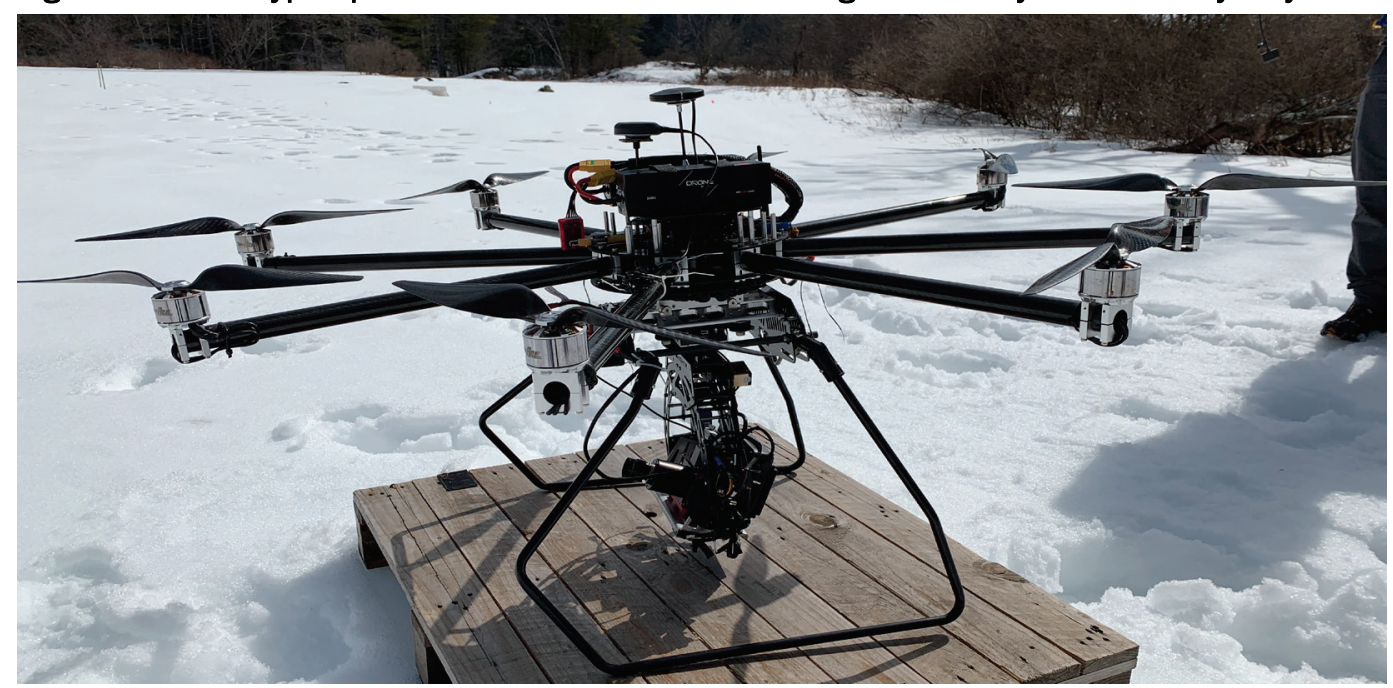

The imager was paired with an SBG Systems Ellipse-N inertial measurement system (INS) with an integrated single antenna real-time kinematic (RTK) Global Navigation Satellite Systems (GNSS) to account for the roll, pitch, and yaw of the aircraft and the geographic position of the imager. The Ellipse- $\mathrm{N}$ is hard-mounted to the hyperspectral imager through a Resonon-supplied bracket known as a "gimbal caddy." A flight computer is also attached via the gimbal caddy that operates the Pika L, Ellipse-N, and the external SSD where the hyperspectral data is recorded. The Pika L was secured to the Infinity 9 via a gimbal that was locked to the yaw of the UAS to stabilize the sensor against the roll and pitch of the UAS during flight. The Pika L was oriented with its lens pointed directly nadir as to avoid the effects of an off-nadir viewing geometry. The GNSS antenna 
puck was placed on a small, carbon-fiber platform elevated above the top of the UAS to allow for the clearest signal and separation from potential sources of interference onboard the aircraft.

In addition to the hyperspectral imager and airborne hardware, a 2.4- $\mathrm{x}$ 2.4-m dual reflectance (12\% and $24 \%$ ) calibration tarp was provided by Resonon to allow for the conversion of the imagery from radiance to reflectance during post-processing of the imagery. The mean spectral response of one of the sides of the calibration tarp (e.g. 24\% reflectance) was calculated in Spectronon Pro and used to correct the radiance imagery for a given flight to reflectance.

\subsection{Flight planning}

The hyperspectral flight plan for the winter data collections was constructed to ensure that each of the sample plots were contained within at least one of the scans. The flight lines were arranged such that each pass was antiparallel to the previous line (e.g. A to A') with a horizontal overlap of $30 \%$ of the total swath width. The line spacing (LS) between adjacent lines was then determined using the percent of overlap between adjacent scans $\left(\mathrm{P}_{\mathrm{o}}\right)$ and the known swath width $(\mathrm{SW})$ :

$$
L S=\left(1-P_{o}\right)(S W)
$$

The first two data acquisitions at the UVD field site (01/26/21 and 02/07/21) had a mission altitude of $50 \mathrm{~m}$ resulting in a ground-swath width of $15.48 \mathrm{~m}$. The ground-swath width can be derived from equation 4 using the mission altitude of a given flight $(\mathrm{h})$ and the FOV of the lens in degrees. Subsequent flights were conducted at an altitude of $60 \mathrm{~m}$ resulting in a swath width of $18.57 \mathrm{~m}$ to ensure that each plot was captured completely within a given scan line.

$$
S W=2 *(h)\left(\tan \frac{F O V^{\circ}}{2}\right)
$$

A rectangular region of interest (ROI) around the 75- x 50-m flight box was created in Google Earth and exported as a keyhole markup language (KML) in order to trigger the automatic data collection. The Pika $\mathrm{L}$ is designed to begin collecting data upon entering a region of interest, as there is no remote control of imager once it is airborne. 
Two sets of calibrations were completed prior to the first data acquisition flight in order to account for the physical offsets between the GNSS antenna, INS, and imager (lever-arm correction) and the calibration of the internal INS magnetometer. The lever arm offsets (X, $\mathrm{Y}$, and $\mathrm{Z}$ offsets) between the GNSS antenna and the INS, as well as between the INS, and the Pika L were measured prior to the magnetic calibration. The convention for measuring all lever-arm distances is as follows: positive $\mathrm{X}$ values are in the direction of flight (forward), positive $Y$ values are to the right of the UAS with respect to the forward direction of travel, and positive $Z$ values are measured downward relative to the top of the UAS. All lever-arm distances were measured with the sensor in its flight position with the lens pointing nadir.

After the determination of the lever arm offsets, a two-dimensional magnetometer calibration was conducted. The internal magnetometer of the Ellipse- $\mathrm{N}$ is used to acquire heading information about the direction of travel of the INS and is sensitive to electromagnetic interference (EMI) from the operation of the UAS. The unique EMI profile of the UAS must be accounted for through the magnetometer calibration, which allows the magnetometer to ignore the diagnostic EMI signature from the UAS when determining an accurate heading.

All flights were conducted near solar noon between 1100 and 1400 in order to minimize the effect of low solar-illumination angles on the spectral response of the surface. Previous work by Nolin and Dozier (1993) demonstrated that there is a significant reduction in snow reflectance for solar-incidence angles of greater than $30 \mathrm{deg}$. This can potentially overestimate grain size, as NIR reflectance decreases with increasing grain size. The 2.4-m calibration tarp was placed near the center of each flight box directly adjacent to one of the sample plots to ensure that the tarp was captured by at least one scan per flight. Data collections were conducted under either uniformly sunny conditions or constant cloud cover so as to maintain consistent illumination conditions during a given flight.

\subsection{Dimensionality reduction}

The high number of spectral channels associated with hyperspectral imagery presents a challenge to the training and performance of machine algorithms due to what is commonly known as the "curse of dimensionality" (Windrim et al. 2019). The high number of spectral bands drastically increases the number of data points, which in turn can 
dramatically increase the computational requirements to train a given machine learning algorithm. Additionally, the large number of features (spectral bands) in a hyperspectral data set relative to the number of unique labels or target values can result in reduced algorithm performance (Windrim et al. 2019). In order to efficiently train robust regression algorithms, it is imperative to either select only the optimum combinations of bands or to represent the most pertinent information in fewer dimensions while preserving most of the variance within the data set. Fortunately, a large number of dimensionality reduction techniques exist that have shown the capability to accomplish this task.

Dimensionality reduction techniques serve two primary purposes: to improve the training speed through the reduction of the total number samples and to remove noise that is often captured in the higher dimensional space (Rodarmel and Shan 2002; Wang and Chang 2006; Villa et al. 2009; Luo et al. 2016). Most dimensionality reduction methods seek to transform the high number of spectral bands into a new set of bands that preserve the most pertinent information in fewer bands. These new bands also have a higher SNR in the lower dimensions (e.g. PCA bands 1 through 10) that decreases in the higher dimensions. For example, Rodarmel and Shan (2002) demonstrated that, after from PCA band 10 onward, the imagery is characterized entirely by noise.

Adjacent hyperspectral bands often contain highly redundant information due to the very narrow spectral resolution that can slow algorithm training (Luo et al. 2016). This redundancy can be diminished through dimensionality reduction techniques, as the total variation between neighboring channels is preserved by transforming the information contained in each band into a lower dimensional subspace whereby the most salient features in the dataset are retained but at a much lower computational cost.

We compared three different dimensionality reduction techniques and their performance on an unsupervised segmentation task using tripod-based hyperspectral imagery collected in winter 2020. The three techniques we compared were PCA, Minimum Noise Fraction (MNF), and Independent Component Analysis (ICA). PCA seeks a linear combination of the hyperspectral bands such that the most PCA seeks a linear combination of the hyperspectral bands such that the most variance lies along the first principal component axes ( $\mathrm{PC} 1$ ) with the second principal component axes 
holding the second most variance, and so forth (Rodarmel and Shan 2002). The original hyperspectral bands that contribute the most to the total variance captured along PC 1 will have a higher signal-to-noise ratio (SNR) and, therefore, will yield better algorithm performance. From visual comparison of PCA results on our winter hyperspectral data set, PCA bands greater than band four contain a significant amount of noise, so only the first four PCA bands were used to train the regression models.

MNF is conceptually similar to PCA in that the linear combinations of the original bands are used to transform the data so that most of the information can be represented in lower dimensional space (Green et al. 1988). MNF ranks the resultant components (transformed bands) by the normalized SNR, instead of ranking the components by the total variance captured along a given component axis as in PCA (Green et al. 1988; Luo et al. 2016). ICA is commonly employed in signal processing for the separation of a given signal into its constituent components and has be applied to hyperspectral data as means of unmixing signal sources (Villa et al. 2009). Whereas PCA and MNF operate using primarily second order statistics, ICA incorporates higher order statistical information while seeking to make each component as independent as possible (Wang and Chang 2006).

All three algorithms were implemented in Python and tested on the same set of imagery captured from Keweenaw Research Center (KRC) using the same Pika L imager configured for terrestrial collection. This imagery was collected on a mixed ice, asphalt, and packed snow surface to assess the instrument's ability to parse these distinct surfaces. Figure 6 shows all three dimensionality reduction techniques produced continuous clusters that aligned closely with actual boundaries of the three surface types. The three techniques produce very similar results in the K-Means clustering tasks, although PCA was the quickest algorithm to converge. Based upon the results of the K-Means clustering task, it was decided that PCA would be used given its comparable performance to the other two techniques and its increased speed. 
Figure 6. Results of K-means data reduction test on data collected from KRC. Each color represents a different "class" or "cluster" identified by the K-means algorithm.

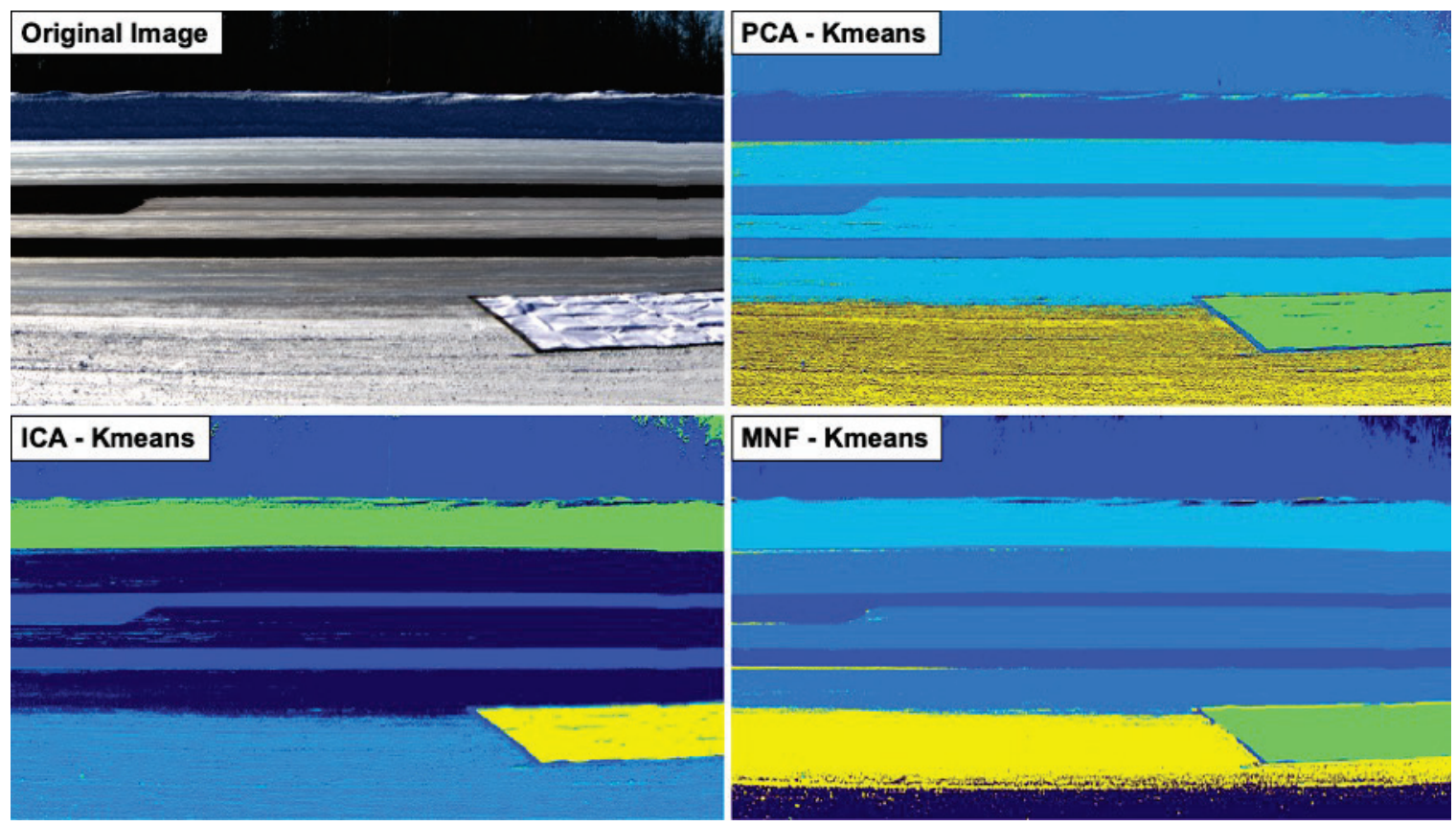

\subsection{Hyperspectral data processing}

The raw imagery recorded by the Pika $L$ needs to be converted to radiance using the 17-mm lens radiometric calibration file supplied by Resonon. This conversion was handled onboard the UAS by uploading the calibration file onto the flight computer. The resulting radiance imagery was then converted to reflectance with the proprietary Spectronon Pro software using the mean spectra obtained from the dual-reflectance calibration tarp. The reflectance data cubes were then converted to fourband PCA data cubes as described in the dimensionality reduction section. The PCA data cubes were then orthorectified in Spectronon Pro using the photogrammetry-derived DEM and then converted to four-band GeoTIFFS (Figure 7). Given the relatively high spatial resolution of the hyperspectral imagery to the number of physical measurements (one per plot), we constrained the orthorectified imagery to $0.1 \mathrm{~m}$ resolution during the orthorectification step, to retain neighborhood information while accounting for the coarser sampling resolution. We then manually cropped each sample plot from their respective four-band PCA GeoTIFF and assigned the corresponding in-situ grain size, liquid water content, and density measurements for each plot as raster layers. 
Figure 7. The corrected reflectance hyperspectral data before (left) and after (right) orthorectification. For scale, the white region in the image is the calibration tarp.

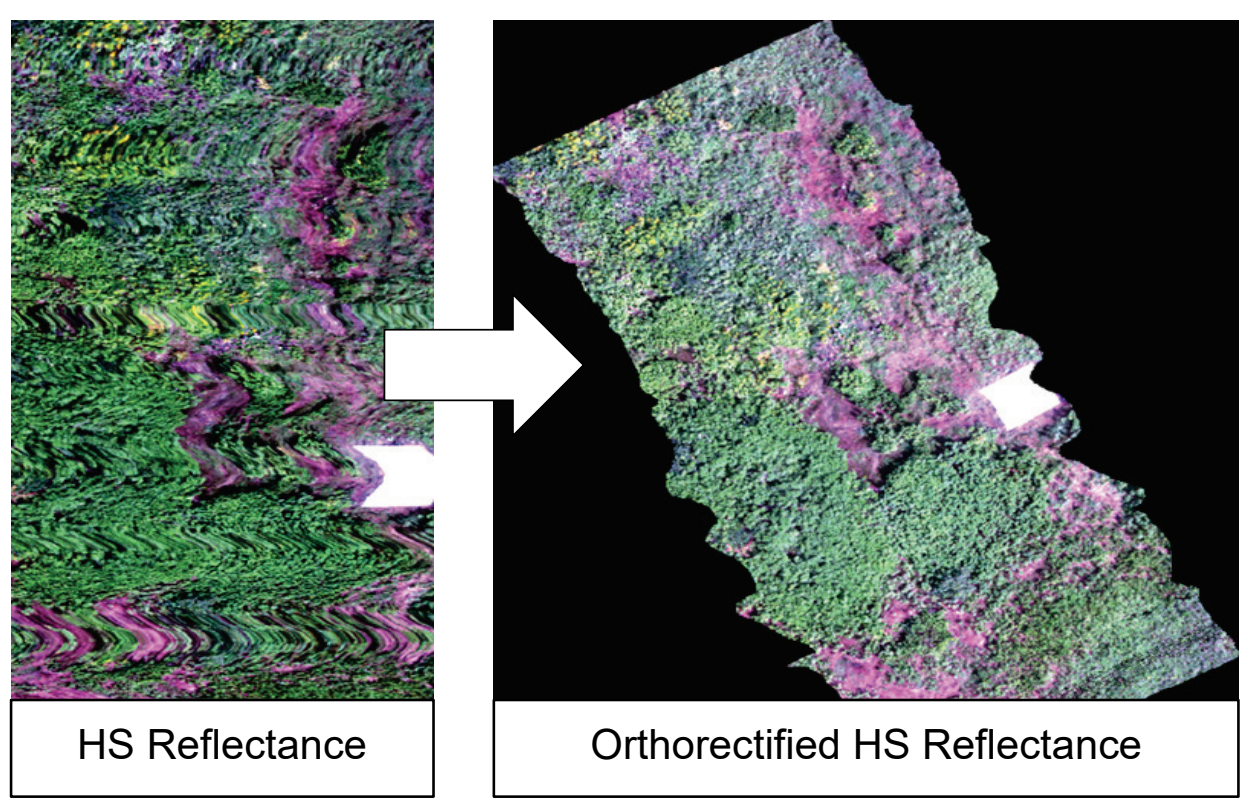

\subsection{Photogrammetry and orthorectification}

To post process the hyperspectral data, it must be orthorectified using a DEM of the sampled region. Since hyperspectral data was collected in the winter and the snow depth varied throughout the season, a DEM needed to be generated for each collection. This was accomplished through the use of photogrammetry. Photogrammetry derives elevation changes using overlapping photos calibrated to established ground control points (Miller et al. 2000). Metashape software was used to generate high-density point clouds through matching features between overlapping aerial photos. Aerial images were collected in an overlapping gridded pattern using a Skyraider UAS platform (Figure 8) with a high-resolution camera. The resultant DEM covered the entire area of hyperspectral imagery at a $\sim 10 \mathrm{~cm}$ resolution. 
Figure 8. Skyraider UAS platform with high resolution camera used for collecting photogrammetric images.

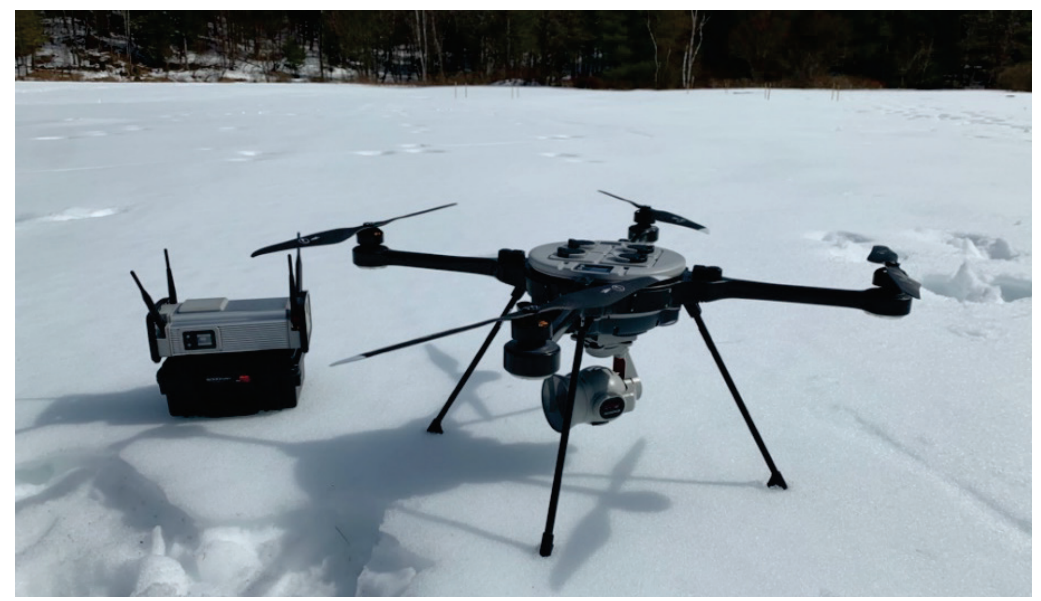

\subsection{Spectroradiometer}

The spectral range of the hyperspectral camera used in this work covered only the visible and beginning of the NIR wavelengths. Since many of the previous studies identified useful peaks in the NIR and shortwave infrared (SWIR) regions, a field spectroradiometer was used during the first field collection to compare with the hyperspectral data (Figure 9). The spectroradiometer was manufactured by Malvern Panalytical Ltd. The instrument captures a spectral range of 350 to $2500 \mathrm{~nm}$ with 3-8 nm of spectral resolution. A 5 -deg field of view foreoptic lens was used. The spectral reflectance from the snow surface at nadir and the spectral reflectance at nadir from a white Spectralon reference plate were measured at each site. The spectral reflectance was calculated by dividing the surface measurement by the reference standard measurement.

Figure 9. Using the field spectroradiometer on the white Spectralon reference plate.

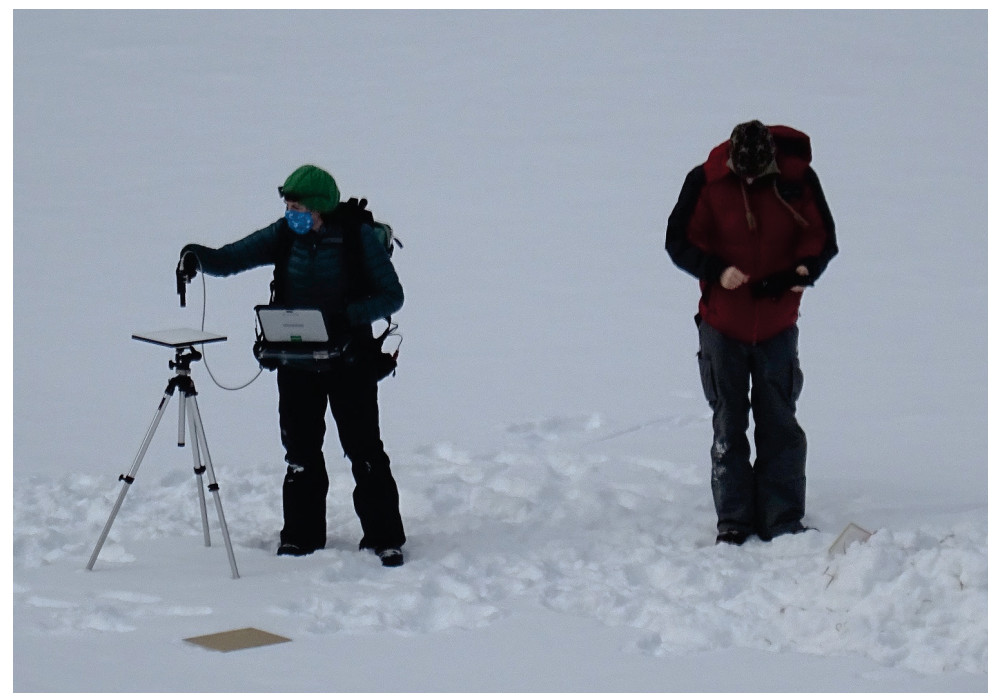




\section{Machine Learning Frameworks}

\subsection{Labeling the data}

In order to train and test a machine learning framework, combinations of input variables are labeled with the appropriate target variables. For this analysis the input variables included the four PCA-derived bands from the hyperspectral imager and the target variables included the density, moisture, and grain size of the snow. The data labeling was done manually using ArcGIS 10.7. First the input bands were clipped to the regions of the test plots (discussed in section 2.1). Then the bands were combined into a single raster where each pixel contained the information of each input band. Finally, each pixel was assigned target variable labels based on the in-situ measurements associated with that particular site on a given collection day. The format of the labeled output file can be seen in Table 1, where the total number of rows is equal to the sum of pixels within all of the test sites over all of the collection days. With roughly 1000 pixels per test site, five test sites, and five collection days the total number of labeled samples was $\sim 25,000$.

Table 1. Example format of input text file for machine learning frameworks.

\begin{tabular}{|c|c|c|c|c|c|c|}
\hline PCA Band 1 & PCA Band 2 & PCA Band 3 & PCA Band 4 & Density & Grain Size & Moisture \\
\hline$A$ & $B$ & $C$ & $D$ & $X$ & $Y$ & $Z$ \\
\hline
\end{tabular}

\subsection{K-Nearest Neighbors (KNN)}

The KNN algorithm uses feature similarity to predict the values of new data points. Essentially, new data points are assigned a value based on how closely they resemble the points in the training dataset (Fix and Hodges 1952). To determine the predicted value of the new point, the graphed distance between the new point and each training data point is calculated. The " $K$ " nearest points are then used to calculate a predicted value based on the average of those points. In this analysis, the KNN algorithm from Python's Scikit-Learn library was used as it provides welltested and easy to implement functionality (Pedregosa et al. 2011). The default hyperparameters were used to build the model, and no parameter tuning was conducted as model results were adequate. 


\subsection{Decision trees}

Decision trees are one of the most commonly used machine learning frameworks for regression. Decision trees are predictive models that use sequential partitioning of data to calculate a target value (Laurent and Rivest 1976). The trees begin with partitioning the "parent" or "root" node using a decision boundary. These partitioned nodes can then split using new features to build a new decision boundary. This creates a tree-like structure, where the end result is a target value that is based on the variable input features. The classification process steps each data point through the tree, making decisions based on its input features until a terminal node (or "leaf") is reached. This terminal node assigns that data point a specific target classification. For this study, the default hyperparameters were used for implementing the random forest framework with the Scikit-Learn library.

\subsection{Support vector regression}

Support vector regression (SVR) is a statistical method that examines the relationship between several input variables (Cortes and Vapnik 1995). SVR is similar to support vector machines in that it is based on the concept of fitting hyperplanes. These hyperplanes are essentially the line of best fit through the training data. This line is determined by a cost function that weights the graphed distance away from each training data point. To make sure outliers are not heavily weighted in the hyperplane fitting, an error value is specified. This value determines the amount of error acceptable in the model and will find an appropriate hyperplane to best fit the data. SVR has been shown to be one of the most accurate regression tools, and one with the lowest computational cost for training the model. A radial basis function was used for the kernel of the SVR model, while the remaining hyperparameters used the standard values from Scikit-Learn.

\subsection{Random forest}

Random forest regression is a supervised learning subtype of decision trees. This algorithm is an ensemble learning method that combines predictions from multiple decision trees to make a more accurate prediction than a single tree (Ho 1995). Each tree is built in isolation from one another with no information being shared between individual trees. Target variable values are predicted for each tree, then the average for each target variable is calculated and used as the final predicted value. Random forest models 
typically outperform decision trees as the parallel structure of the forests minimizes overfitting that can commonly occur within decision trees. For this study, the default hyperparameters were used for implementing the random forest framework with the Scikit-Learn library.

\subsection{Gradient boosted trees}

Gradient boosting is another supervised machine learning subtype of decision trees. The model is built through combining an ensemble of "weak learner" trees into a "strong learner." The trees are sequentially built with each iteration giving added weight to mislabeled data from the iterations before it (Friedman 1999a; Friedman 1999b). Through this process, high accuracies can be achieved. However, gradient boosted trees can be easily overfit if the model hyperparameters are not used to limit the size of the trees. Common hyperparameters include maximum leaves, which limit the number of target variables allowed per tree, and maximum depth, which limits the number of decision nodes within a tree. The default hyperparameters in the Scikit-Learn library are designed to limit overfitting for most models. Thus, the default parameters were used to train the regression framework for this analysis.

\subsection{Artificial Neural Networks}

Artificial Neural Networks (ANNs) are widely used for a variety of machine learning tasks including regression (Higham and Higham 2019). ANNs can serve as universal function approximator for any continuous function and can map highly non-linear relationships due to the use of activation functions at each node of the network (Higham and Higham 2019). This allows ANNs to operate as a "black box" in regard to the learned parameters that are leveraged in the final regression algorithm. Unique, fully connected feedforward ANN models were developed for each of the three properties of interest, grain size, density, and liquid water content for a total of three separately trained networks.

Each training step through the models is conducted on a pixel-by-pixel basis, as each pixel in a data cube can be represented as vector where the number of rows corresponds to the spectral bands or channels. All three models use a rectified linear unit as the activation functions within each hidden layer. We employed the Adam optimizer to update the weights in the network following each training batch, as Adam has shown to result in faster and more efficient convergence in comparison to the stochastic 
gradient descent method (Kingma and Ba 2014). The ANN models were developed using the Tensorflow, Keras, and SciKit-Learn Python libraries.

Hyperparameter selection and optimization is critical to the development of effective ANN models (Feurer and Hutter 2019). The model hyperparameters for the neural networks evaluated in this study include the number of hidden layers and number of neurons per hidden layer. The ANN model hyperparameters were determined using a random grid search methodology where a range of possible values for each hyperparameter was supplied, and then random combinations of these hyperparameter values were evaluated after training was completed. The random grid search algorithm was implemented using 3 -fold cross-validation with ten different iterations (hyperparameter combinations) for a total of 30 different data and model combinations. The optimum set of hyperparameters for each model as determined through the random grid search are presented in Table 2.

Table 2. Artificial neural network model hyperparameters. ANN Model: physical property used to train model; Input Neurons: number of nodes in input layer; Hidden Layers: number of hidden layers in each network; Neurons: number of neurons in each hidden layer.

\begin{tabular}{|l|c|c|c|}
\hline \multicolumn{1}{|c|}{ ANN Model } & Input Neurons & Hidden Layers & Neurons \\
\hline Grain Size & 4 & 3 & 26 \\
\hline Density & 4 & 3 & 26 \\
\hline Moisture & 4 & 3 & 28 \\
\hline
\end{tabular}

In order to prevent the overfitting of our ANN models to the training data, we implemented an early stopping protocol that stops training if the validation score stops decreasing over 20 epochs. Each network was run for 100 training epochs or until the early stopping criteria was met.

\subsection{Stacked regression}

Stacked regression is an ensemble machine learning framework that uses a meta-learning algorithm to best combine the predictions from several other machine learning algorithms (Breiman 1996). In this study, we used all of the "traditional" (non-ANN) machine learning algorithms as model inputs. The idea behind stacking models is that it can use the strengths of each input predictive model while minimizing their weaknesses. Essentially, if there are several predicted values for a specific data point (one from each base model), the predictions can be averaged to increase accuracy. Increased accuracy is not always 
guaranteed with model stacking, as some model predictions may cause outlier predictions, rendering the stacked framework a poorer performer than individual base models. Stacking is often used for classification examples, but the same theory applies to continuous datasets. The stacked regression framework in Scikit-Learn was used with the default hyperparameters to test the efficacy of this approach. 


\section{Results}

\subsection{Model performance}

Initially, we focused on evaluating the traditional machine learning algorithms. These included: KNN, SVR, decision tree, random forest, gradient boosted regression, and the stacked regression framework. We chose to use Mean Absolute Error (MAE) as our performance indicator, as it is a commonly used metric for determining the accuracy of a regression models (Figure 10). This metric was also used to help identify appropriate hidden layers and neurons for the ANN. Beginning with grain size as the predicted variable, $\mathrm{KNN}$ and random forest regression had the lowest MAE, while the stacked model had the highest. KNN had the smallest standard deviation of MAE values, and decision trees had the largest. When looking at the moisture content target variable, once again $\mathrm{KNN}$ and random forest seemed to have the lowest MAE, while stacking the models had the worst performance and largest standard deviation. Finally, for the density target variable, the KNN and random forest had the lowest MAE while stacking the models produced the worst results. In this instance, SVR had the tightest spread of data, while decision trees and stacking had the widest spread. Overall, it is clear that $\mathrm{KNN}$ and random forest produce the best results for each target variable. It is surprising that the stacked regression framework produced the lowest accuracy results. However, this is likely because noise is being introduced into the stacked framework from the poor performance of each individual model. A more in-depth analysis into the tuning of model hyperparameters may help increase the accuracy of the stacked framework.

The ANN models performed similarly to the other regression algorithms aside from the poorly performing stacked regressor. The moisture model exhibited the largest MAE relative to the range of values of the three models, likely due to the high number of near-zero liquid water content samples. The snow moisture model performance is likely to improve for all machine learning (ML) frameworks with an increased number of relatively wet snow samples. Increased sampling during the late winter and early spring would provide more wet snow endmembers that should improve the model performance. Despite the reduced performance of the ANN model on the moisture data, the data collections from 11 March 2021 show pockets of near-surface snow melt that prove the capability of NIR hyperspectral imagery to capture surface snow melt (Figure 11). 
Figure 10. Boxplots showing each ML model's performance (MAE) when predicting snow grain size, moisture content, and density.
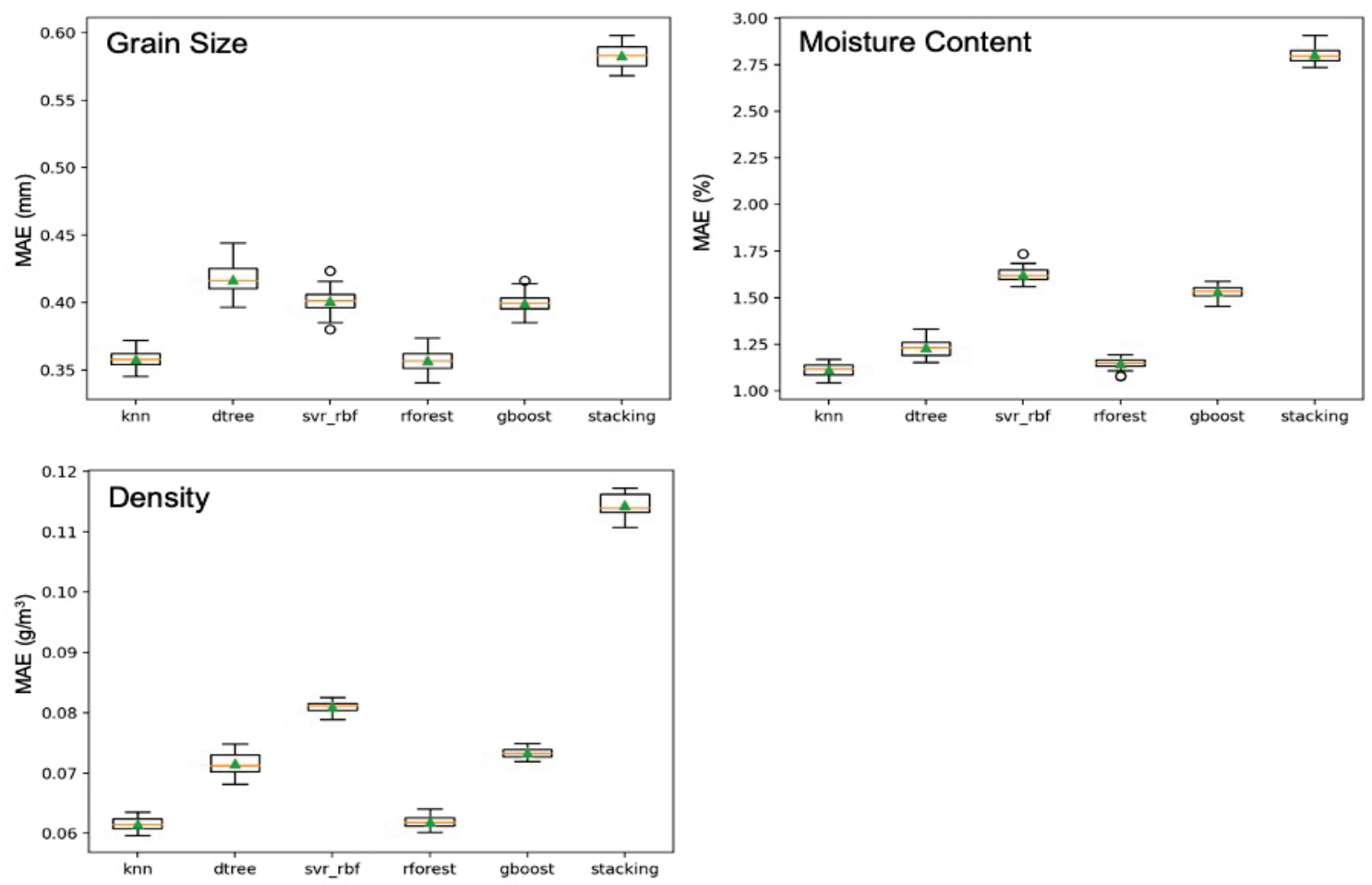

The grain size models likewise produced reasonable results but the MAE was still relatively large in comparison to the lower range of grain size values. The largest source of error is likely in the grain size card measurement technique as it assigns a discrete estimate of grain size rather than a range of values. The grain size measurement technique employed here likely produces an overestimate of mean grain size since the larger grains of a given sample are more visible leading to a bias towards larger grain size estimation. One solution would be to use chilled grain sieves to more robustly assign grain size distributions to sample plots that would require the binning of samples into grain size classes based upon the dominant grain size. 
Figure 11. Orthomosaic image collected on $3 / 11 / 21$ when the snow had wet pockets forming near the surface; associated hyperspectral composite composed primarily of the NIR bands; and the predicted moisture using the hyperspectral false color composite as the input data into the KNN model framework.
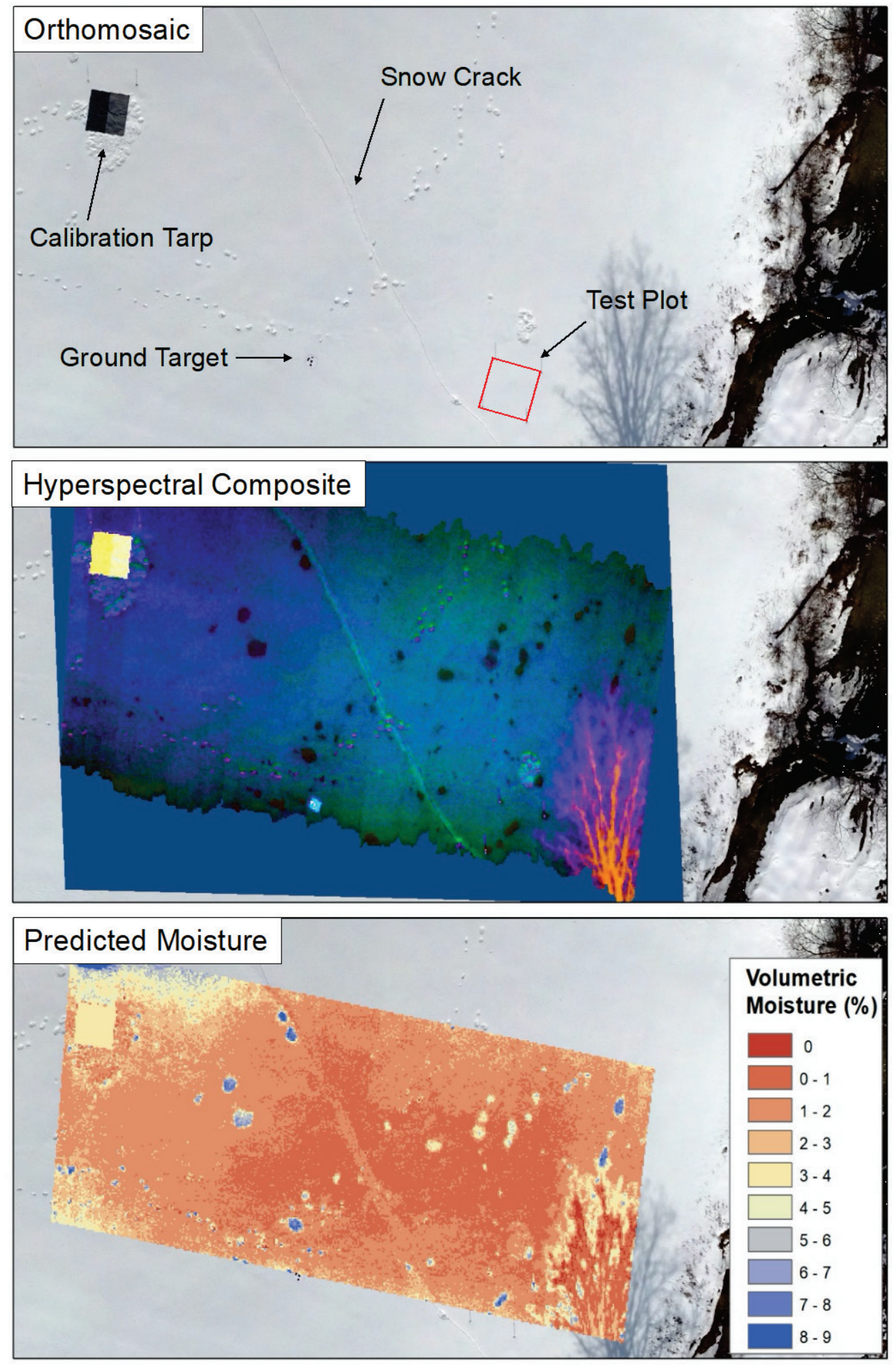
The snow density models performed similarly well to the other two models. The MAE for the density models was lower $\left(\sim 0.06 \mathrm{~g} / \mathrm{cm}^{3}\right)$ relative to the full range of observed density values $\left(0.160-0.535 \mathrm{~g} / \mathrm{cm}^{3}\right)$. Unlike the moisture measurements, the snow density measurements showed greater variation between sites and between sampling days, yielding a more continuous range of values to train the models upon. In comparison to grain size, both moisture and density are bulk measurements rather than a discrete value that represents the approximate average of a random group of grains that may or may not be totally representative of the true mean. Furthermore, the precision associated with both density and moisture measurements are higher than the grain size measurements, which are likely to be rounded to the nearest half-millimeter. More precise measurements of snow grain size would likely lead to improved algorithm performance.

Future developments to these models should incorporate SWIR data (900 $\mathrm{nm}-1800 \mathrm{~nm}$ ) in addition to VIS/NIR since it is likely that including SWIR band information would greatly improve model performance. Snow reflectance exhibits greater sensitivity to changes in grain size at wavelengths greater than $1000 \mathrm{~nm}$, which is beyond the spectral range of the Pika L (Nolin and Dozier 1993; Nolin and Dozier 2000; Dozier and Painter 2004). The addition of SWIR band information as well as wider range of snow conditions will serve to only bolster the performance of the algorithms presented here.

\subsection{Correlations between physical and mechanical measurements}

The response of passive-sensing instruments such the Pika L depend upon the illumination conditions as well as the optical properties of the surface being imaged. In order to robustly link sensor performance with sensor response, it is critical that a relationship be established between the features known to affect surface reflectance (e.g. grain size) and the mechanical properties of the surface. A series of pairwise Pearson correlation coefficients were calculated for each of the in-situ physical measurements and the mechanical measurements collected across the winter field season at each plot (Figure 12). There is a positive relationship between the three physical properties (grain size, density, and snow moisture), which aligns with the field observations that wettest snow conditions also coincided with the densest and coarsest grain snow. The mechanical properties do not strongly correlate with any of the physical properties, which is indicative of the more complex relationship between the physical properties of snow and the 
mechanical properties. The surface roughness of the snow, which was not considered here, likely influences the coefficient of friction of the surface. The addition of a neighborhood metric such as moving standard deviation, may provide a stronger relationship with coefficient of friction. Future work should look to add textural variables about the snow surface conditions that incorporate neighborhood information to better delineate the changes in surface friction.

Figure 12. Pearson correlation matrix comparing relationships between in-situ physical and mechanical snow properties.

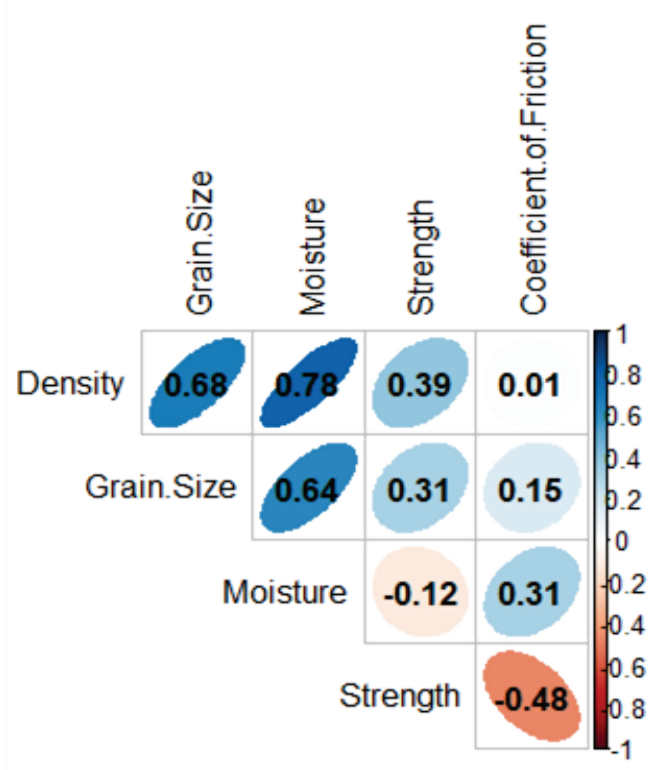

The limited correlation between the strength of the snow pack and the insitu physical properties is most likely due to the fact that optical penetration depth of snow is limited to the first few centimeters of the snow pack (Nolin and Dozier 2000). Strength values will likely vary through a depth profile as snow pack characteristics vary drastically. Snow grain size often increases with increasing depth, which might lead to a stronger medium than would be apparent from surface characterization, with the exception of depth hoar that has more faceted grains. Grain size alone may also not be a dominant control on snow strength as the interaction between the sizes, shapes, and cohesion of the grains within the snow pack likely contribute to the bulk strength of the snow. Density typically correlates well to strength, but only for dry, finegrained snow. Thus, more research is needed to evaluate the interplay between grain size, grain shape, and grain sorting on the mechanical response of snow to quantify the impact of each property. 


\section{Conclusions}

The aim of this study was twofold. The primary goal was to train several machine learning frameworks to predict surface snow properties using airborne hyperspectral data. The predictions were validated against in-situ measurements of grain size, density, and moisture content. The secondary goal was to identify relationships between the physical properties that the models were trained to predict and mechanical properties of the snow. The following are the main takeaways from this analysis:

- Overall, the individual machine learning models had better performance than the stacked model framework.

- The best-performing (lowest MAE) traditional machine learning frameworks were the random forest and $\mathrm{KNN}$ models.

- Density and grain size had the lowest MAE relative to measured value ranges, likely due to the lack of moisture ranges measured during collections.

- ANN seemed to perform as well as the traditional machine learning frameworks, but has added training complexity.

- Hyperspectral bands were strongly weighted in the principal component bands, likely due to the strong correlation to snow microstructure properties.

- Density, grain size, and moisture content all had a statistically significant positive correlation to one another.

- Density and grain size had a slightly positive correlation to compressive strength, while moisture had a much weaker negative correlation.

- Moisture had a slightly positive correlation to the surface friction, likely due to the surface being slightly lubricated by the wet snow.

The results of this analysis show great promise for using airborne hyperspectral imagery to map surface snow properties. Being able to automatically predict snow characteristics at high spatial and temporal scale will be critical for ensuring successful off-road autonomous vehicle mobility in winter conditions. 


\section{Recommendations}

This work provides a preliminary assessment showcasing the efficacy of using aerial hyperspectral imagery to predict surface snow properties for autonomous mobility purposes. While there are reasonable predictions presented by the models in this work, more in-situ measurements must be collected over more variable snow conditions. For moisture content, this can be accomplished by sampling more frequently during the melt season in the spring. Better characterization of grain size will also be important to provide a more continuous distribution of data as opposed to the discrete values estimated from this work. This can be achieved through the use of grain size sieving instead of visual identification on a grain size card. Hyperspectral cameras characterize only the surface of the snow pack, therefore information of snow properties at depth must be characterized using another sensor such as radar, or through sequential hyperspectral scans of one region between snow storm events. More robust correlations must be made between the physical and mechanical properties as well. This will be more fruitful in a laboratory environment where the parameters can be more easily controlled and replicated. If robust correlations between the physical and mechanical properties are identified, then those relationships can be used in tandem with the machine learning framework presented here to predict strength and friction coefficients of the surface snow pack. 


\section{References}

Breiman, L. 1996. Stacked regressions. Machine Learning 24(1):49-64.

Cortes, C., and V. Vapnik. 1995. Support-vector networks. Machine Learning 20(3):273297.

Denoth, A., A. Foglar, P. Weiland, C. Mätzler, H. Aebischer, M. Tiuri, and A. Sihvola. 1984. A comparative study of instruments for measuring the liquid water content of snow. Journal of Applied Physics 56(7):2154-2160.

Dozier, J., and T. H. Painter. 2004. Multispectral and hyperspectral remote sensing of alpine snow properties. Annual Review of Earth and Planetary Sciences 32.

Ewing, J., T. Oommen, P. Jayakumar, and R. Alger. 2020. Utilizing hyperspectral remote sensing for soil gradation. Remote Sensing 12(20):3312.

Feurer, M., and F. Hutter. 2019. Hyperparameter optimization. In Automated machine learning, 3-33). Cham, Switzerland: Springer.

Fix, E., and J. L. Hodges Jr. 1952. Discriminatory analysis-nonparametric discrimination: Small sample performance. California University Berkeley.

Friedman, J. H. 1999a. Greedy function approximation: a gradient boosting machine. Department of Statistics, Stanford University techinical report. San Francisco, CA: Stanford University. . 1999b. Stochastic gradient boosting. Department of Statistics, Stanford University technical report. San Francisco, CA: Stanford University.

Green, A. A., M. Berman, P. Switzer, and M. D. Craig. 1988. A transformation for ordering multispectral data in terms of image quality with implications for noise removal. IEEE Transactions on Geoscience and Remote Sensing 26(1):65-74.

Gusain, H. S., A. Singh, A. Ganju, and D. Singh, D. 2004. Characteristics of the seasonal snow cover of Pir Panjal and Great Himalayan ranges in Indian Himalaya. In Proceedings ISSMA-2004, 97-102. Manali.

Haq, M. A., A. Ghosh, G. Rahaman, and P. Baral. 2019. Artificial neural network-based modeling of snow properties using field data and hyperspectral imagery. Natural Resource Modeling 32(4):e12229.

Higham, C. F., \& Higham, D. J. 2019. Deep learning: An introduction for applied mathematicians. Siam review, 61(4), 860-891.

Ho, T. K. 1995. Random decision forest. In Proceedings of the 3rd international conference on document analysis and recognition, 14-16 August, Vol. 1416, p. 278282. Montreal, Canada. 
Hodgdon, T. S., A. J. Fuentes, J. L. Olivier, B. G. Quinn, and S.A. Shoop. 2021. Automated terrain classification for vehicle mobility in off-road conditions. ERDC/CRREL TR-20-220. Hanover, NH: U.S. Army Engineer Research and Development Center.

Kingma, D. P., and J. Ba. 2014. Adam: A method for stochastic optimization. In Proceedings of the International Conference of Learning Representations, April 14-16. Banff, AB, Canada.

Laurent, H., and R. L. Rivest. 1976. Constructing optimal binary decision trees is NPcomplete. Information processing letters 5(1):15-17.

Luo, G., G. Chen, L. Tian, K. Qin, and S. E. Qian. 2016. Minimum noise fraction versus principal component analysis as a preprocessing step for hyperspectral imagery denoising. Canadian Journal of Remote Sensing 42(2):106-116.

Miller, D. R., C. P. Quine, and W. Hadley. 2000. An investigation of the potential of digital photogrammetry to provide measurements of forest characteristics and abiotic damage. Forest Ecology and Management 135:279-288.

Nolin, A. W., and J. Dozier. 1993. Estimating snow grain size using AVIRIS data. Remote Sensing of Environment 44(2-3):231-238. . 2000. A hyperspectral method for remotely sensing the grain size of snow. Remote Sensing of Environment 74(2):207-216.

Parker, M., B. Quinn, J. Bates, T. Hodgdon, M. Bodie, S. Shoop, and A. Stott. 2021. Exploring cold regions autonomous operations. Journal of Terramechanics 96: 159-165.

Pedregosa, F., G. Varoquaux, A. Gramfort, V. Michel, B. Thirion, O. Grisel, and E. Duchesnay. 2011. Scikit-learn: Machine learning in Python. Journal of Machine Learning Research 12:2825-2830.

Perla, R. 1969. Strength tests on newly fallen snow. Journal of Glaciology 8(54):427-440.

Rodarmel, C., and J. Shan. 2002. Principal component analysis for hyperspectral image classification. Surveying and Land Information Science 62(2):115-122.

Selige, T., J. Böhner, and U. Schmidhalter. 2006. High resolution topsoil mapping using hyperspectral image and field data in multivariate regression modeling procedures. Geoderma 136(1-2):235-244.

Shekhar, C., S. Srivastava, H. S. Negi, and M. Dwivedi. 2019. Hyper-spectral data based investigations for snow wetness mapping. Geocarto International 34(6):664687.

Singh, S. K., G. Jain, A. Siddiqui, S. Naik, B. P. Rathore, V. Garg, and J. Dozier. 2019. Characterization and retrieval of snow and urban land cover parameters using hyperspectral imaging. Current Science 116(7):1182.

Villa, A., J. Chanussot, C. Jutten, J. A. Benediktsson, and S. Moussaoui. 2009. On the use of ICA for hyperspectral image analysis. In IEEE International Geoscience and Remote Sensing Symposium, Vol. 4, pp. IV-97. Piscataway, NJ: IEEE. 
Wang, J., and C. I. Chang. 2006. Independent component analysis-based dimensionality reduction with applications in hyperspectral image analysis. IEEE Transactions on Geoscience and Remote Sensing 44(6):1586-1600.

Windrim, L., R. Ramakrishnan, A. Melkumyan, R. J. Murphy, and A. Chlingaryan. 2019. Unsupervised feature-learning for hyperspectral data with autoencoders. Remote Sensing 11(7):864.

Zhang, H. 2016. Surface characterization techniques for polyurethane biomaterials. In Advances in polyurethane biomaterials, 23-73). Sawston, UK: Woodhead Publishing. 


\section{Acronyms and Abbreviations}

\begin{tabular}{|c|c|}
\hline ANN & Artificial Neural Network \\
\hline CRREL & Cold Regions Research and Engineering Laboratory \\
\hline DEM & Digital Elevation Model \\
\hline EMI & Electromagnetic Interference \\
\hline ERDC & U.S. Army Engineer Research and Development Center \\
\hline FOV & Field of View \\
\hline GNSS & Global Navigation Satellite System \\
\hline ICA & Independent Component Analysis \\
\hline IFOV & Instantaneous Field of View \\
\hline KML & Keyhole Markup Language \\
\hline KNN & K-Nearest Neighbors \\
\hline $\mathrm{KRC}$ & Keweenaw Research Center \\
\hline MAE & Mean Absolute Error \\
\hline $\mathrm{MNF}$ & Minimum Noise Fraction \\
\hline NIR & Near Infrared \\
\hline PCA & Principal Component Analysis \\
\hline RGB & Red, Green, Blue \\
\hline ROI & Region of Interest \\
\hline SVR & Support Vector Regression \\
\hline SWIR & Short Wave Infrared \\
\hline UAS & Unmanned Aerial System \\
\hline UAV & Unmanned Aerial Vehicle \\
\hline UVD & Union Village Dam \\
\hline VIS & Visible \\
\hline
\end{tabular}




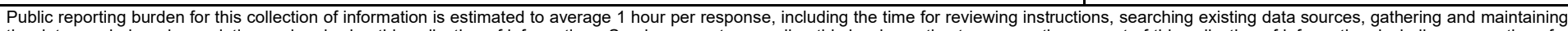

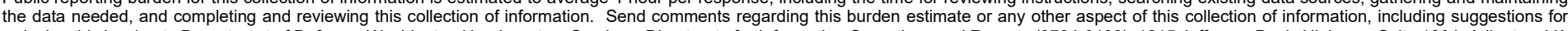

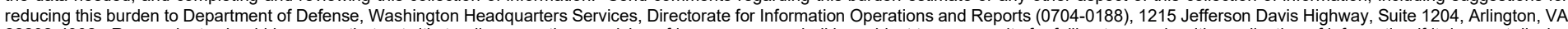

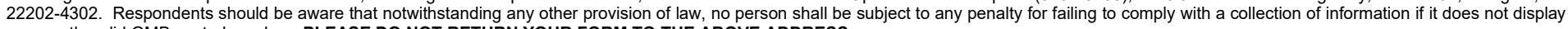
a currently valid OMB control number. PLEASE DO NOT RETURN YOUR FORM TO THE ABOVE ADDRESS.
1. REPORT DATE (DD-MM-YYYY)
2. REPORT TYPE
3. DATES COVERED (From - To)

September 2021

Final

\section{TITLE AND SUBTITLE}

Characterizing Snow Surface Properties Using Airborne Hyperspectral Imagery for

Autonomous Winter Mobility

\section{AUTHOR(S)}

Taylor S. Hodgdon, Anthony J. Fuentes, Brian G. Quinn,

Bruce C. Elder, and Sally A. Shoop

\section{5a. CONTRACT NUMBER}

5b. GRANT NUMBER

5c. PROGRAM ELEMENT NUMBER 0603463A

5d. PROJECT NUMBER

BP4

5e. TASK NUMBER

SBP402

5f. WORK UNIT NUMBER

2686F6

7. PERFORMING ORGANIZATION NAME(S) AND ADDRESS(ES)

8. PERFORMING ORGANIZATION REPORT NUMBER

U.S. Army Engineer Research and Development Center (ERDC)

Cold Regions Research and Engineering Laboratory (CRREL)

ERDC/CRREL TR-21-14

72 Lyme Road

Hanover, NH 03755-1290

9. SPONSORING / MONITORING AGENCY NAME(S) AND ADDRESS(ES)

10. SPONSOR/MONITOR'S ACRONYM(S)

Headquarters, U.S. Army Corps of Engineers

Washington, DC 20314-1000

11. SPONSOR/MONITOR'S REPORT NUMBER(S)

\section{DISTRIBUTION / AVAILABILITY STATEMENT}

Approved for public release; distribution is unlimited.

\section{SUPPLEMENTARY NOTES}

Terrain Conditions Forecasting Program

\section{ABSTRACT}

With changing conditions in northern climates it is crucial for the United States to have assured mobility in these high-latitude regions. Winter terrain conditions adversely affect vehicle mobility and, as such, they must be accurately characterized to ensure mission success. Previous studies have attempted to remotely characterize snow properties using varied sensors. However, these studies have primarily used satellite-based products that provide coarse spatial and temporal resolution, which is unsuitable for autonomous mobility. Our work employs the use of a UAV mounted hyperspectral camera in tandem with machine learning frameworks to predict snow surface properties at finer scales. Several machine learning models were trained using hyperspectral imagery in tandem with in-situ snow measurements. The results indicate that random forest and k-nearest neighbors models had the lowest Mean Absolute Error for all surface snow properties. A Pearson correlation matrix showed that density, grain size, and moisture content all had a significant positive correlation to one another. Mechanically, density and grain size had a slightly positive correlation to compressive strength, while moisture had a much weaker negative correlation. This work provides preliminary insight into the efficacy of using hyperspectral imagery for characterizing snow properties for autonomous vehicle mobility.
15. SUBJECT TERMS
Snow
Hyperspectral

Autonomy
Mobility
Remote sensing

\section{SECURITY CLASSIFICATION OF:}

\section{a. REPORT}

Unclassified

\section{c. THIS PAGE}

Unclassified 17. LIMITATION
OF ABSTRACT

SAR

\section{Snow-Measurement}

Snow mechanics-Remote sensing

Drone aircraft

Hyperspectral imaging

\section{NUMBER 1 19a. NAME OF RESPONSIBLE} OF PAGES

39

\section{PERSON}

19b. TELEPHONE NUMBER (include area code) 